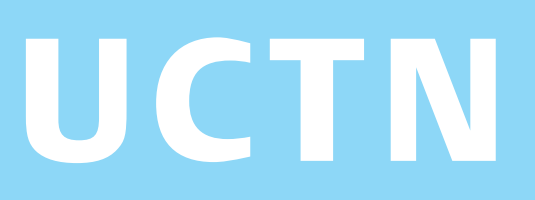

\title{
Acute abdominal pain caused by a prolapsed duodenal polyp that resolved when the polyp broke off spontaneously
}

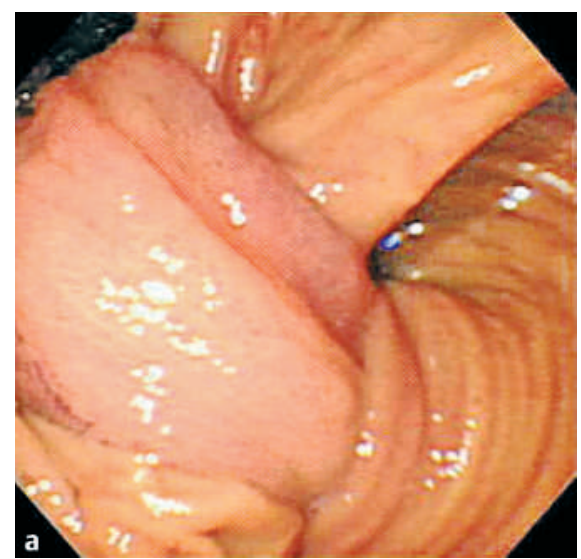

A 48-year-old woman presented with a 1day history of acute abdominal pain after a bout of drinking-related vomiting. She had no significant medical history or family history and was not taking any medications. There were no abnormal findings on careful physical examination, and the standard laboratory results were also within normal ranges. Upper gastrointestinal endoscopy revealed a long stalk arising from the duodenal bulb that had prolapsed into the distal portion of the duodenum (Figure $\mathbf{1} \mathbf{a}$ ). The scope could not be advanced more distally because the stalk was impacted in the duodenal lumen. A small-bowel series showed a large polypoid mass with a long stalk (Figure 1 b ).

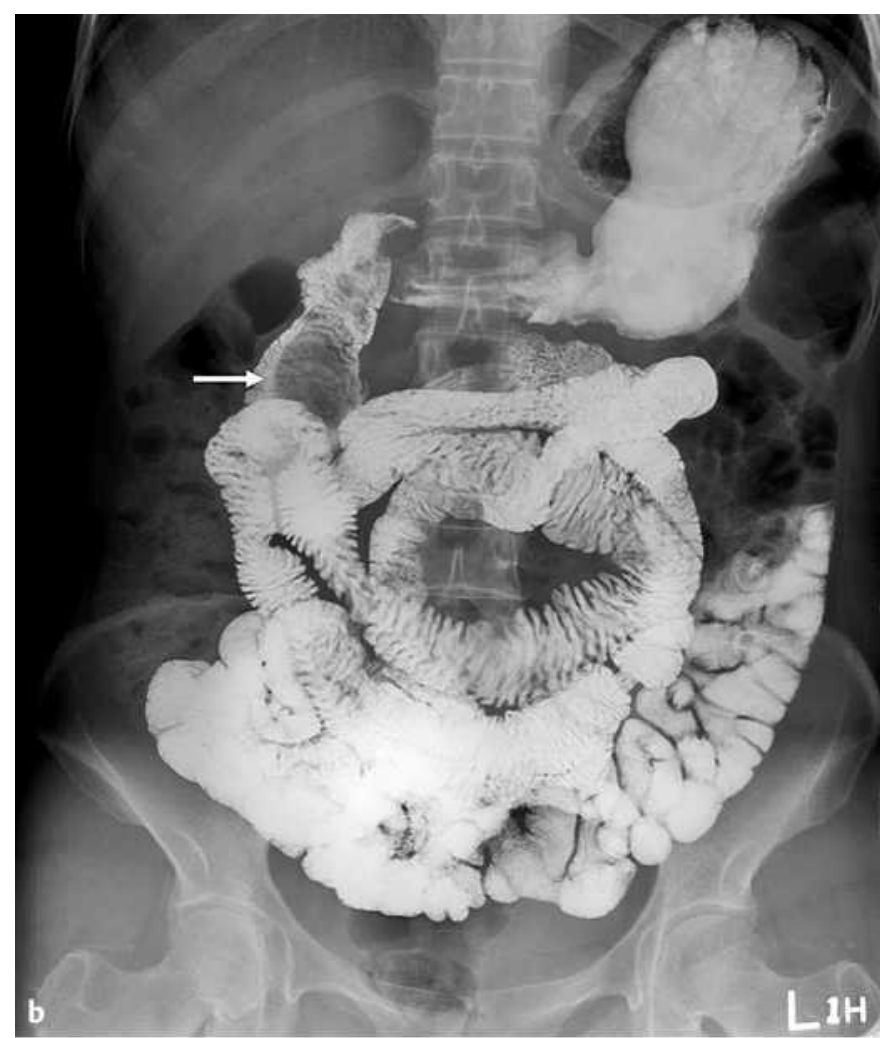

Figure 1 a Endoscopic view of a long stalk that was prolapsing into the distal portion of the duodenum. b Smallbowel series showing a large pedunculated polyp in the second portion of the duodenum (arrow).

On day 3 the abdominal pain disappeared spontaneously. Follow-up upper gastrointestinal endoscopy revealed only an edematous cut-off stalk with ulceration at its tip in the duodenal bulb (Figure $\mathbf{2}$ a). Follow-up small-bowel series also showed no evidence of the previously noted mass (Figure $\mathbf{2} \mathbf{b}$ ). Histopathologic evaluation of the biopsy specimen taken from the remnant of the stalk revealed inflammatory exudates with granulation tissue. The patient is still being followed up and has not complained of any unusual symptoms to date. There have been previous case reports describing compression and obstruction due to a prolapsed polyp as a cause of acute abdominal pain [1-2].

Endoscopy_UCTN_Code_CCL_1AB_2AZ_3AB

\section{K. J. Kim', G. H. Lee ${ }^{2}$}

${ }^{1}$ Department of Gastroenterology, Kosin University Gospel Hospital, Kosin University College of Medicine, Busan, South Korea

2 Department of Gastroenterology, Asan Medical Center, University of Ulsan College of Medicine, Seoul, South Korea. 


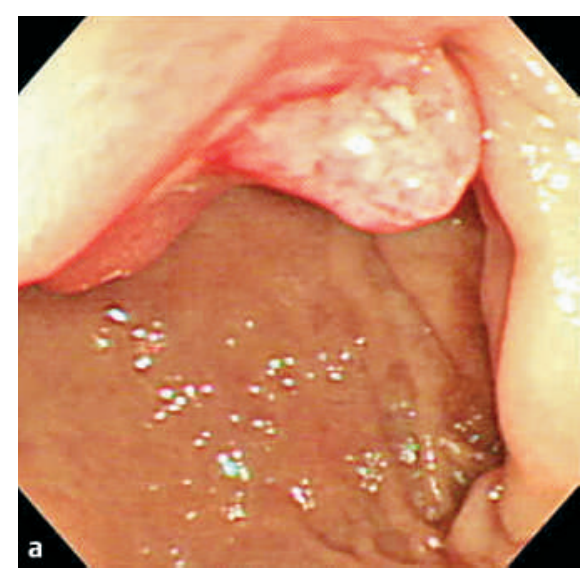

\section{References}

${ }^{1}$ Gencosmanoglu R, Sen-Oran E, Kurtkaya-Yapicier O, Tozun N. Antral hyperplastic polyp causing intermittent gastric outlet obstruction: case report. BMC Gastroenterol 2003; 3: 16

2 de la Cruz RA, Albillos JC, Oliver JM et al. Prolapsed hyperplastic gastric polyp causing pancreatitis: case report. Abdom Imaging 2001; 26: $584-586$

\section{Corresponding author}

\section{K. J. Kim, MD}

Department of Gastroenterology Kosin University Gospel Hospital Kosin University College of Medicine 34 Amnam-dong

Seo-gu

Busan 602-702

South Korea

Fax: $\quad+82-51-990-5206$

Email: drkkj@paran.com

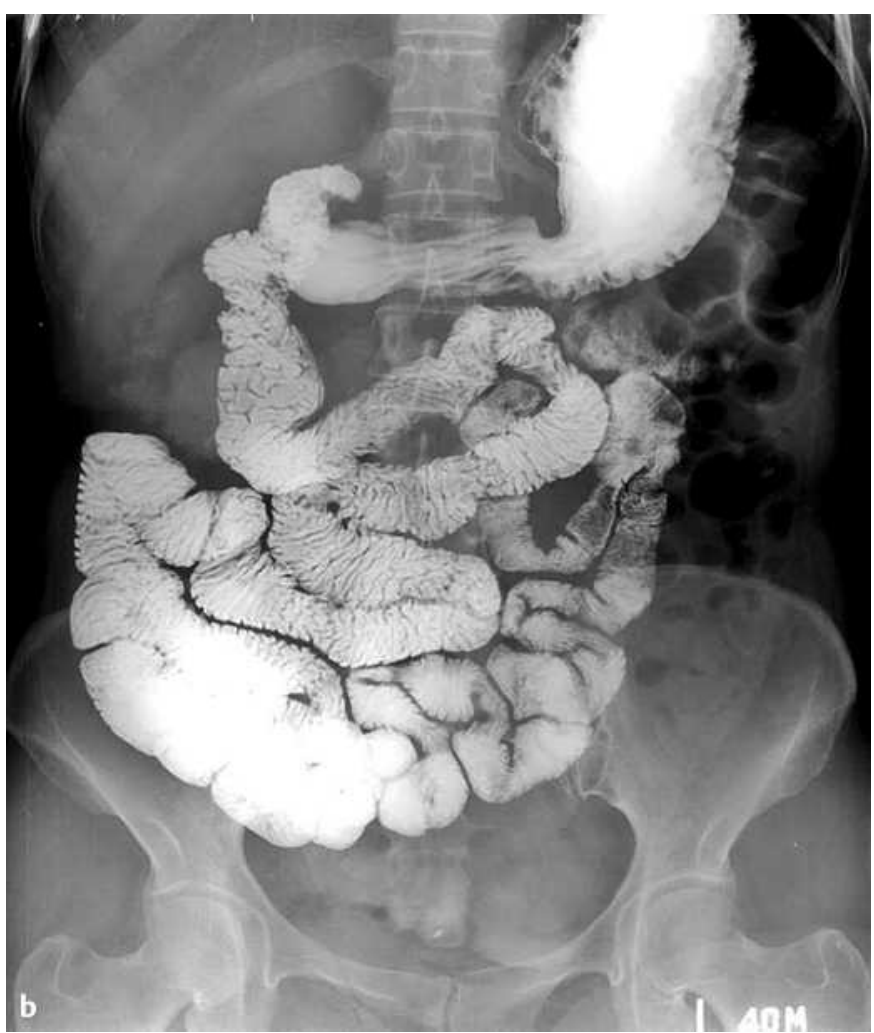

Figure 2 a Endoscopic view of an edematous and ulcerated stalk stump at the duodenal bulb. $\mathbf{b}$ The followup small-bowel series showed no evidence of the previously noted polypoid mass. 